\title{
Bilateral Supratentorial Subdural Hematomas after Craniotomy for Posterior Fossa Tumor Removal
}

\author{
Aikaterini Solomou ${ }^{1}$ Pantelis Kraniotis ${ }^{1}$ George Bonanos ${ }^{2}$ Constantine Constantoyannis ${ }^{2}$ \\ 1 Department of Radiology, MRI Unit, University Hospital of Patras, Greece \\ 2 Department of Neurosurgery, University Hospital of Patras, Greece \\ Address for correspondence Aikaterini Solomou, PhD, University \\ Hospital of Patras, Department of Radiology, MRI Unit, Leof. \\ Ippokratous 26500 Rion, Greece (e-mail: solomou@med.upatras.gr).
}

J Neurol Surg Rep 2018;79:e23-e25.

\begin{abstract}
Keywords

- posterior fossa tumor

- hemangiopericytoma

- chronic subdural hematoma

A 69-year-old man was admitted to the emergency department with headache and dizziness. He was submitted to brain computed tomography (CT) which showed a tumor in the right cerebellar hemisphere, findings which were subsequently confirmed with magnetic resonance imaging (MRI). He underwent a paramedian suboccipital craniotomy for removal of the mass. Histology confirmed the presence of a hemangiopericytoma. The patient was discharged 5 days postoperatively with improvement in his symptoms. Fifteen days later, he presented with gait difficulties. Clinical examination revealed positive Mingazzini sign on his left side. He was submitted to brain MRI which revealed bilateral subdural hematomas on late subacute stage with mass effect and midline shift caused by the largest on the right. The patient underwent burr hole evacuation of the right subdural hematoma. The postoperative CT showed evacuation of the right chronic subdural hematoma. Two days postoperatively, the patient's symptoms improved.
\end{abstract}

\section{Case Report}

A 69-year-old Caucasian male, fit and well, apart from a wellcontrolled hypertension, was admitted to the emergency department with gradually worsening headache and dizziness, dating 30 days back prior to admission. The neurological examination revealed mild dysmetria of the right hand. The blood tests and the electrocardiogram were unremarkable. The brain computed tomography (CT) showed a right cerebellar mass.

He was subsequently submitted to brain magnetic resonance imaging (MRI) for the further evaluation of the mass which was mainly solid with smaller cystic areas. The lesion caused mass effect onto the surrounding cerebellar parenchyma and to the fourth ventricle (-Fig. 1). The patient underwent a paramedian suboccipital craniotomy for tumor removal. Because of high tumor vascularity, massive bleeding occurred during the operation. The mass was completely removed. The immediate postoperative period was unremarkable and the patient was discharged after a few days with symptoms improvement. Histology confirmed the presence of a hemangiopericytoma.

received

July 13, 2017

accepted after revision

January 22, 2018
Fifteen days postoperatively, the patient presented with gait disturbances. He was submitted to brain MRI, which showed bilateral hematomas, larger one on the right, causing mass effect with midline shift and a smaller one on the left. The signal characteristics were compatible with late subacute hematomas. A fluid-fluid level was present on the right ( - Fig. 2A-C). The patient underwent burr hole evacuation of the right subdural hematomas. The postoperative CT showed complete evacuation of the right hematoma and no enlargement of the left one. The symptoms improved and patient was eventfully discharged.

There was no clinical evidence of interim development of postoperative intracranial hypotension. Moreover, there were no findings suggestive of intracranial hypotension on MRI (-Fig. 3).

\section{Discussion}

Cerebellar tumors are usually removed by midline or paramedian suboccipital approach. The most common postoperative complications are cerebrospinal fluid leak, leading to pseudomeningocele, adipose graft prolapse into

\footnotetext{
(c) 2018 Georg Thieme Verlag KG Stuttgart · New York
}

License terms

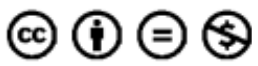




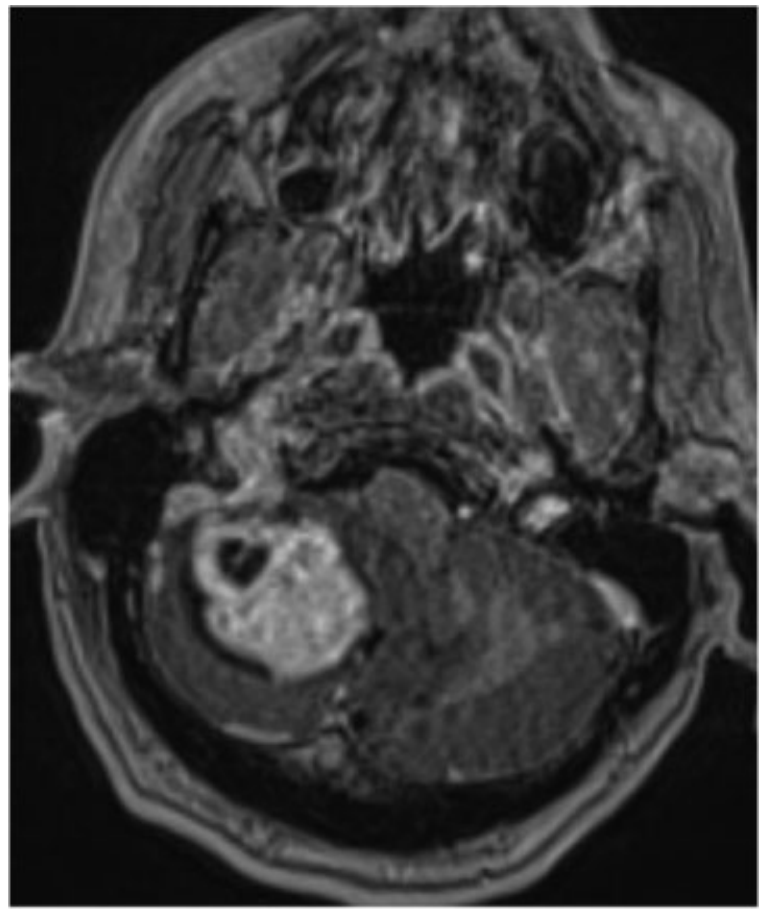

Fig. 1 Axial postcontrast T1-weigheted image. The mass within the right cerebellar hemisphere shows mainly solid components and smaller cystic areas as well. The solid component enhances fairly homogeneously. The lesion causes mass effect changes in the surrounding parenchyma.

cerebellopontine angle, vascular-mechanical cranial nerves IX and X or brain-stem injuries, intracranial abscess or bone flap osteomyelitis, ischemic or hemorrhagic brain-stem stroke, facial paralysis, and sensory deficits from injury to cranial nerves $\mathrm{V}$ and VII and ataxia from cerebellar edema. The incidence of postoperative hematomas, far from the surgical bed, is very low ranging between $0.8 \%$ and $1.3 \%{ }^{1,2}$ They are usually located in the supratentorial compartment and are either ipsilateral or contralateral ${ }^{3,4}$ to the surgical bed.

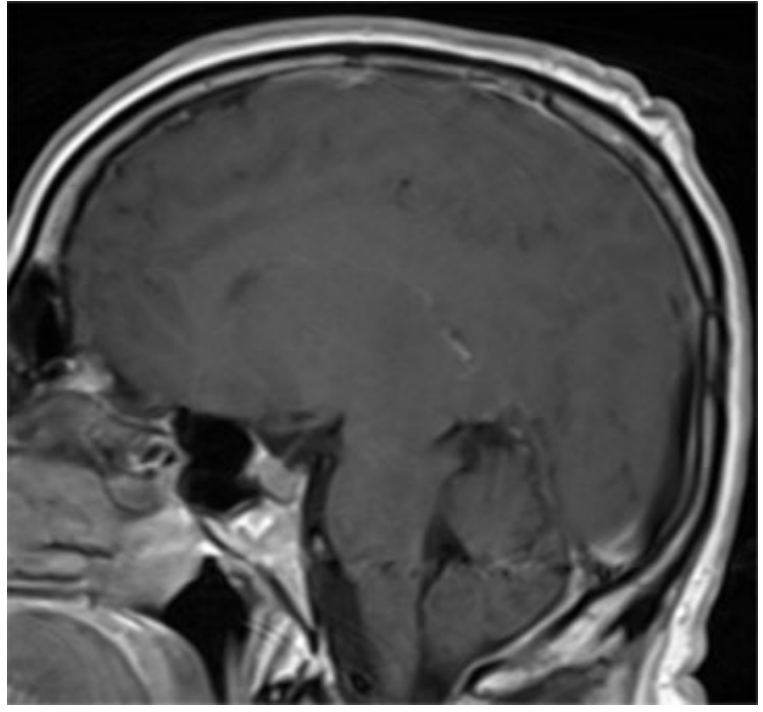

Fig. 3 Sagittal postcontrast T1-weighted image. The suprasellar and prepontine cisterns are within normal limits. There is no flattening of the pons or medulla. Furthermore, there is no diffuse pachymeningeal enhancement.

Nevertheless, hematomas occurring across the tentorium are very rare, and so far only a few cases are found in the English literature. ${ }^{5-7}$

Extradural hematomas are known postoperative complications caused by the stripping of dura from the bone, after craniotomy; another cause may be constant bleeding from the bone marrow, which is injured by the pins of the clamps ${ }^{1}$ Hematomas remote to the surgical bed is more perplex, particularly when they happen contralaterally to the surgical site.

Postcraniotomy hematomas may be caused by coagulopathy $^{8,9}$ injury to subdural or extradural bridging veins, ${ }^{10}$ breach of the inner cortex by the head clamp pins ${ }^{1,4,6}$ insufficient dural hitches, ${ }^{1,10}$ and by hemodynamic changes during the operation. ${ }^{11}$

Extradural hematomas after posterior fossa surgery are reported in five cases, caused probably lowering of the

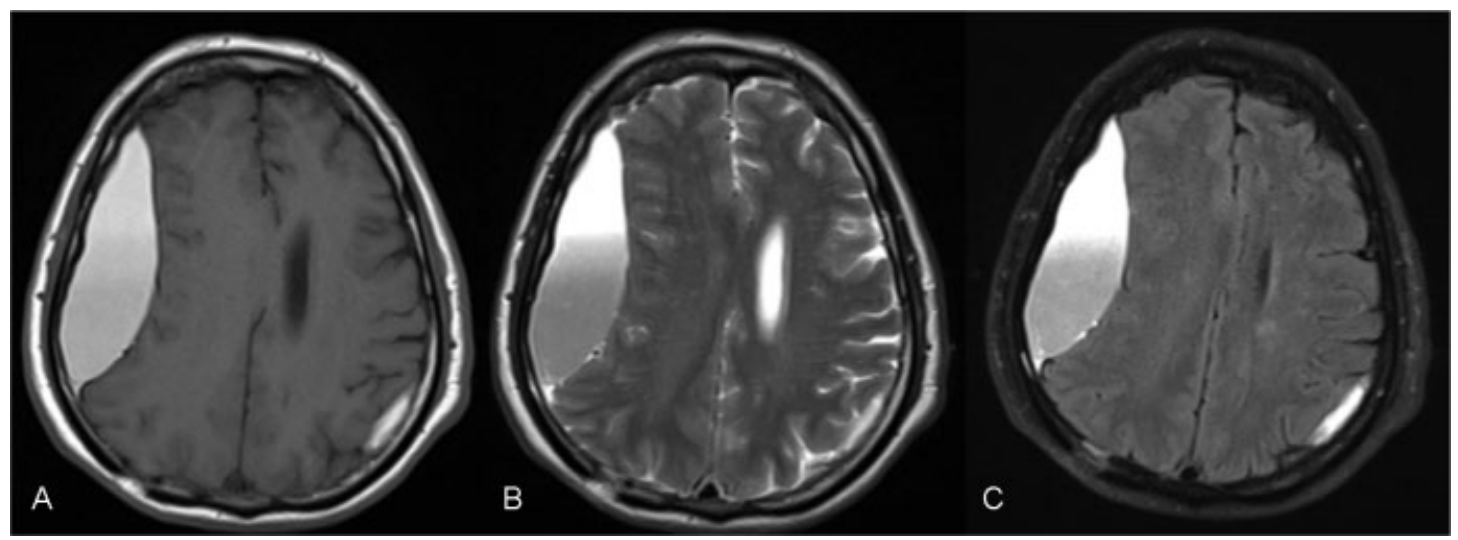

Fig. 2 (A-C) Axial T1-weighted image, T2-weighted image, and fluid-attenuated inversion recovery showing bilateral subdural hematomaslarger one on the right and a smaller one on the left. The signal characteristics are in keeping with late subacute hematomas. A fluid-fluid level is found on the right. 
ventricular pressure. ${ }^{1}$ Penetrating pins from the head fixation clamps is another known cause of hematomas. 6,10

A more recent case has also reported the development of extradural hematoma on the one side and a concomitant contralateral subdural hygroma following posterior fossa surgery. $^{12}$

However, in our case we stress the development of bilateral subdural supratentorial hematomas, after posterior fossa surgery. It is interesting to note that in both cases the larger subdural hematoma is ipsilateral to the surgical site.

In conclusion, unilateral supratentorial subdural hematomas, after craniotomy for the excision of a posterior fossa tumor, are extremely rare. Bilateral subdural supratentorial hematomas have not been previously reported in the English literature, to our knowledge.

\section{References}

1 Jan M, Gouaze A, Elie A, Lapierre F, Santini JJ. [Extra dural hematoma complicating ventricular decompression during posterior fossa exploration (author's transl)]. Neurochirurgie 1978; 24(02):137-139

2 Tsugane R, Sugita K, Sato O. [Supratentorial extradural hematomas following posterior fossa craniectomy (author's transl)]. No Shinkei Geka 1976;4(04):401-403
3 Driesen W, Elies W. Epidural and subdural haematomas as a complication of internal drainage of cerebrospinal fluid in hydrocephalus. Acta Neurochir (Wien) 1974;30(1-2):85-93

4 Wolfsberger S, Gruber A, Czech T. Multiple supratentorial epidural haematomas after posterior fossa surgery. Neurosurg Rev 2004;27(02):128-132

5 Mohindra S, Savardekar A, Luthra A. Supratentorial hematoma during infratentorial surgery: a short series of three cases. Indian J Neurosurg 2012;1:155-157

6 Pandey P, Madhugiri VS, Sattur MG, Devi B I. Remote supratentorial extradural hematoma following posterior fossa surgery. Childs Nerv Syst 2008;24(07):851-854

7 Tondon A, Mahapatra AK. Superatentorial intracerebral hemorrhage following infratentorial surgery. J Clin Neurosci 2004;11 (07):762-765

8 Fujimoto Y, Aguiar PH, Carneiro JD, et al. Spontaneous epidural hematoma following a shunt in an infant with congenital factor $\mathrm{X}$ deficiency. Case report and literature review. Neurosurg Rev 1999;22(04):226-229

9 Vrettou CS, Stavrinou LC, Halikias S, et al. Factor XIII deficiency as a potential cause of supratentorial haemorrhage after posterior fossa surgery. Acta Neurochir (Wien) 2010;152(03):529-532

10 Kalkan E, Eser O. Supratentorial intracerebral haemorrhage following posterior fossa operation. Neurol India 2006;54(02):220-221

11 Bucciero A, Quaglietta P, Vizioli L. Supratentorial intracerebral hemorrhage after posterior fossa surgery. Case report. J Neurosurg Sci 1991;35(04):221-224

12 Vupputuri R, Rajesh A. Unusual supratentorial complication following posterior fossa surgery. J Neurosci Rural Pract 2014;5 (02):199-201 